\title{
La mise en scène des voix narratives dans la réécriture de la "Belle dame sans mercy" par Anne de Graville
}

\section{Elisabetta Barale}

\section{(2) OpenEdition Journals}

Édition électronique

URL : http://journals.openedition.org/studifrancesi/14583

DOI : $10.4000 /$ studifrancesi. 14583

ISSN : 2421-5856

Éditeur

Rosenberg \& Sellier

\section{Édition imprimée}

Date de publication : 1 décembre 2018

Pagination : 407-415

ISSN : 0039-2944

\section{Référence électronique}

Elisabetta Barale, «La mise en scène des voix narratives dans la réécriture de la "Belle dame sans mercy" par Anne de Graville », Studi Francesi [En ligne], 186 (LXII | III) | 2018, mis en ligne le 01 décembre 2019, consulté le 07 janvier 2021. URL : http://journals.openedition.org/studifrancesi/14583 ; DOI : https://doi.org/10.4000/studifrancesi. 14583

\section{(c)}

Studi Francesi è distribuita con Licenza Creative Commons Attribuzione - Non commerciale - Non opere derivate 4.0 Internazionale. 


\title{
La mise en scène des voix narratives dans la réécriture de la "Belle dame sans mercy" par Anne de Graville
}

\begin{abstract}
This article focuses on Anne de Graville's Rondeaux, a rewriting of Alain Chartier's Belle dame sans mercy, transmitted by the single manuscript Paris, BnF, fr. 2253. Firstly, the analysis of both the layout and the re-elaboration techniques will allow us to observe that the work brings to the stage a plurality of voices, which correspond to socially well-defined characters, according to the interpretation of the rhetorical conception of the "character", theorized in the tenth chapter of the Instructif de seconde rbétorique. Furthermore, a thematic investigation based on some lexical choices will show that a particular attention is reserved to the presence of images evoking the game of roles between the lady and the knight; we will finally advance the hypothesis that the Rondeaux take part in a virtual "collaborative community" diachronically engaged in the rewriting of Chartier's masterpiece.
\end{abstract}

«En maistre Allain de ses oeuvres j'ay quis/ a mon juger le plus fin et exquis» ${ }^{1}$ : c'est avec ces mots qu'Anne de Graville, poétesse à la cour de François ${ }^{\mathrm{er} 2}$, exprimait sa dette envers Alain Chartier ${ }^{3}$ dans la dédicace à la reine Claude figurant dans son remaniement de la Belle dame sans mercy en soixante-et-onze rondeaux, composés vers 1515 et conservés dans le manuscrit unique Paris, $\mathrm{BnF}$, fr. $2253^{4}$. On sait que la Belle dame sans mercy avait déjà suscité plusieurs réactions et que de nombreuses continuations, imitations et traductions avaient vu le jour au fil du temps ${ }^{5}$ : au début du XVI

(1) Ms. Paris, BnF, fr. 2253, f. 2v.

(2) Sur Anne de Graville cfr. Marquis de Laqueuille, Anne de Graville, ses poésies, son exhérédation, Chartres, Garnier, 1858; M. de Montmorand, Une femme poète du XVI siècle. Anne de Graville, sa famille, sa vie, son cuvre, sa postérité, Paris, Picard, 1917; H. LAmArQue, Autour d'Anne de Graville: le débat de la "Dame sans sy" et épitaphe de la poétesse, dans Mélanges sur la littérature de la Renaissance à la mémoire de V. L. Saulnier, éd. P.-G. Castex, Genève, Droz, 1984, pp. 603-611; M.D. OrTH, Dedicating women: manuscript culture in the French Renaissance, and the case of Catherine d'Ambroise and Anne de Graville, «Journal of the Early Book Society of the Study of Manuscript and Printing History», 1997, pp. 17-47; C. Müller, Le rôle de l'intellectuel et l'écriture poétique des femmes dans les cours princières au passage du XV au XVI siècle, dans Courtly Literature and Clerical Culture, Papers from the Triennial Congress of the International Courtly Literature Society, éd. C. Hubert, H. Lähnemann, Tübingen, Attempto, 2002, pp. 221-230; I. Ákerlund, Sixteenth Century French Women Writers: Marguerite d'Angoulême, Anne De Graville, the Lyonnese School, Jeanne De Jussie, Marie Dentière, Camille de Morel, New York, E. Mellen Press, 2003, pp. 45-63.

(3) Sur Alain Chartier cf. R. Meyenberg, À propos d'Alain Chartier. Problèmes d'analyse de textes en prose français, dans Rbétorique et mise en prose au $X V^{\bullet}$ siècle, Actes du Colloque International sur le Moyen Français [Milan, 4-6 mai 1998], éd. S. Cigada, A. Slerca, Milano, Vita e Pensiero, 1991, pp. 27-37; R. Meyenberg, Alain Chartier, prosateur et l'art de la parole au XV siècle. Études littéraires et rbétoriques, Berne, Francke, 1992.

(4) On dispose d'une transcription datant du XIX siècle: "La belle dame sans mercy" en Fransk dik.t forfattad, éd. C. Walhund, Uppsala, Almquist \& Wiksells Boktryckeri-Aktiebolag, 1897. Cf. aussi: G. GuAZZOTTI, "Une femme de lettres" réécrit la "Belle dame sans mercy" d'Alain Chartier un siècle plus tard. Édition critique d'après le manuscrit Paris, Bibl. nationale de France, fr. 2253, mémoire de maîtrise sous la dir. de P. Cifarelli, Università degli Studi di Torino, 2013/2014.

(5) Les ouvrages de Chartier se diffusèrent bientôt grâce à l'imprimerie; de nombreuses réimpressions en furent faites sur la base de la première édition de ses œuvres complètes: Les Fais maistre Alain Chartier notaire et secretaire du Roy Charles VI ${ }^{e}$, Paris, Pierre Le Caron, 1489. En outre, les textes se rattachant à la 
siècle, Chartier était encore réputé comme un maître absolu de l'éloquence. Dans l'Instructif de seconde rhetorique, traité de versification en rime sans doute rédigé dans les années 1460, mais qui jouit d'une diffusion exceptionnelle grâce à l'édition du Jardin de plaisance et Fleur de rhetorique publié par Antoine Vérard en 1501-1502 l'auteur, connu sous le pseudonyme de l'Infortuné ${ }^{7}$, manifeste lui aussi son admiration pour «maistre Alain», dont les œuvres constituent le principe et le fondement de son art poétique ${ }^{8}$. Puisque Anne de Graville semble partager avec l'Infortuné, aussi bien qu'avec les savants de son époque, l'intention d'établir un échange intellectuel avec Chartier, cette contribution se propose de revenir sur l'étude de ses Rondeaux selon une double perspective. En premier lieu, il s'agira de vérifier dans quelle mesure la réécriture semble interpréter la conception rhétorique du «personnage»" théorisée dans le dixième chapitre de l'Instructif; deuxièmement, une analyse thématique s'appuyant sur certains choix lexicaux effectués par Anne de Graville permettra de mettre en rapport les rondeaux avec les remaniements d'autres poètes qui s'engagèrent dans la reprise du chef d'œuvre de "Maistre Alain", en se confrontant les uns aux autres à l'intérieur d'une sorte de «communauté collaborative», dont le modèle socio-culturel, tant historique et synchronique que virtuel et diachronique, a été proposé par E. Cayley ${ }^{10}$.

Dans le dernier chapitre du Jardin de Plaisance, l'Infortuné explique comment construire une forme narrative versifiée ${ }^{11}$ lorsqu'on y fait intervenir des locuteurs différents du narrateur ${ }^{12}$; à cet effet, il introduit la notion de «personnage», en tant qu'individu caractérisé par une condition sociale et une situation déterminées, qui doit posséder un système de valeurs et un niveau de langue appropriés ${ }^{13}$. D'après ce traité, la création poétique se concrétiserait alors dans la mise en scène d'une pluralité de voix et dans le déploiement d'un art de la conversation ${ }^{14}$. La réécriture de la poétesse paraît

Querelle de la Belle dame sans mercy, étudiés autrefois par A. PIAGET, La belle dame sans mercy et les poésies lyriques, Genève, Droz, 1949 ont fait l'objet d'un regain d'intérêt de la part de la critique: cf. The poetical works of Alain Chartier, éd. J. C. Laidlaw, Cambridge, Cambridge University Press, 1974; D. F. Hult, Le cycle de "La Belle dame sans mercy", Paris, Champion, 2003.

(6) Le Jardin de plaisance et Fleur de rhétorique, éd. E. Droz et A. Piaget, 2 vol., Paris, F. Didot, 19101925. Sur le Jardin de plaisance cf. J.H.M. TAYLOR, La mise en mélange au XV" siècle: feuilleter le "Jardin de Plaisance", dans Le goût du lecteur à la fin du Moyen Âge, éd. D. Bohler, Paris, Le Léopard d'Or, 2006, pp. 47-63. Pour une nouvelle hypothèse sur la fonction de l'Instructif dans l'édition de Vérard cf. J.H.M. TAYLOR, La double fonction de l'Instructif de la seconde rbétorique": une bypothèse, dans L'écrit et le manuscrit à la fin du Moyen Âge, éd. T. Van Hemelryck, C. Van Hoorebeeck, Turnhout, Brepols, pp. 343-351.

(7) La Muse et le Compas: poétiques à l'aube de l'âge moderne, éd. J.-C. MonfERran, Paris, Classiques Garnier, 2015, pp. 16-27.

(8) «Par maistre Alain, a qui Dieu pardon face, I cest art icy se monstre et verifie». Cf. ibid., vv. 14861487, p. 119. Sur les arts poétiques: J.-C. Monferran, L'école des muses: les arts poétiques français à la Renaissance (1548-1610): Sébillet, Du Bellay, Peletier et les autres, Genève, Droz, 2011.

(9) La Muse et le Compas cit., p. 41.

(10) Sur la notion de «communauté collaborative» cf. E. J. CAYLEy, Debate and dialogue. Alain Chartier in his cultural context, Oxford, Oxford University Press, 2006; J. MACRAE, A Community of Readers: The Quarrel of the "Belle dame sans mercy", dans A companion to Alain Chartier (c. 1385-1430): Father of French Eloquence, éd. D. Delogu, J. MacRae, E. Cayley, Leiden-Boston, Brill, 2015, pp. 200-222.

(11) Sur le problème terminologique posé par la liste des genres concernés dans le dixième chapitre du traité cfr. C. Thiry, Le théâtre, ou la poétique de l'entredeux, «Études de Lettres» 4, 2002, pp. 43-69, notamment pp. 56-59.

(12) E. Buron, "Faire en personnages". De la théorie de l' Instructif" à la pratique du "Jardin de plaisance", «Cahiers de Recherches Médiévales et Humanistes» 21, 2011, pp. 205-223, notamment pp. 211-212.

(13) «Item, l'on doit donner langaige I a chascun selon la personne». Cfr. La Muse et le Compas cit., pp. 41-45; citation vv. 1981-1982, p. 136.

(14) S.R. Kovacs, Staging lyric performances in early print culture: "Le jardin de plaisance et fleur de rethorique”, «French Studies» 55, 2001, pp. 1-24, notamment pp. 13-14. 
partager cette conception, en faisant du manuscrit un véritable ensemble polyphonique, dans lequel les voix d'Anne de Graville et de Chartier se cachent derrière celles des deux interlocuteurs du débat amoureux. En effet, dans le manuscrit parisien, le remaniement de la poétesse est accompagné des huitains correspondants de Chartier, copiés dans la marge ${ }^{15}$. Ce choix inhabituel de mise en page témoigne de la volonté de la dame d'honneur de placer la voix de «maistre Allain» à côté de la sienne. L'espace de la réécriture métamorphose ainsi les deux auteurs en «personnages» qui se font face dans un jeu lyrique à l'apparence théâtrale ${ }^{16}$. Les rondeaux et les huitains, loin d'être des antagonistes, manifestent une complémentarité sur le plan formel ${ }^{17}$.

La poétesse semble étudier les formules de l'écrivain, en mettant en œuvre plusieurs stratégies destinées à élaborer une construction rhétorique de ses «personnages» fondée souvent sur des procédés de renversement du texte-source. Ceux-ci peuvent résulter en la simple transformation du statut syntaxique de certains énoncés: c'est ainsi que les phrases négatives sont tournées en des constructions affirmatives et vice-versa, que des phrases passives sont réécrites en actives ou bien le contraire et que certains mots sont remplacés par leurs antonymes:

\begin{tabular}{|c|c|}
\hline Alain Chartier, La belle dame sans mercy & Anne de Graville, Rondeaux \\
\hline $\begin{array}{l}\text { [...] Ne sçay que vous appellez "bien" } \\
\text { (mal emprumpte bien aultruy non!) }[\ldots] \\
{\left[\text { h. } 30^{18}, \text { f. } 16 \mathrm{v}\right]}\end{array}$ & $\begin{array}{l}\text { [...] Je le congnois que vous appellez "bien" } \\
\text { et "mal" aussy }[\ldots] \\
\text { [r. } 28, \text { f. 16v }]\end{array}$ \\
\hline $\begin{array}{l}\text { [...] Pitié, Justice et Droit } \\
\text { sont de cueurs de dames bannis }[\ldots] \\
{[\text { h. } 69, \text { f. } 36 \mathrm{r}]}\end{array}$ & $\begin{array}{l}\text { [...] Pitié, Doulceur, Justice et Droit } \\
\text { en cueur de dame ont perdu leur endroit } \\
{[\ldots]} \\
{[\text { r. } 67, \text { f. } 36 r]}\end{array}$ \\
\hline $\begin{array}{l}\text { [...] Quant Pitié qui est endormie } \\
\text { mettroit a mes maulx fin et terme }[\ldots] \\
{[\text { h } 47, \text { f. } 25 \mathrm{r}]}\end{array}$ & $\begin{array}{l}\text { [...] Mais se Pitié qui par raison m'est deue } \\
\text { estoit de vous dessus moy estendue, } \\
\text { j'aurois santé que je n'ay que demye }[\ldots] \\
{[\text { r. } 45, \text { f. } 25 \text { r] }}\end{array}$ \\
\hline
\end{tabular}

Par delà la nécessité de répondre parfois aux exigences de la versification, ces exemples montrent que, tout en restant fidèle au texte de Chartier, Anne de Graville se sert de modifications à première vue minimales, dans le but de renouveler le portrait de la dame et de son amant. Le «je» de la femme s'affirme en soulignant l'importance de la conscience de l'individu (r. 28), tandis que la transformation du bannissement de Pitié, Justice et Droit de son cœur en une perte de la place qu'ils

(15) Dans son étude pionnière, Carl Wahlund a mis en lumière que la version de la Belle dame sans mercy transcrite à côté des rondeaux n'est pas la source directe de la réécriture, car la poétesse se serait servie d'une ou de plusieurs versions différentes des huitains. Cfr. M. BOUCHARD, Les belles [in]fidèles: traduire l'ambiguité masculine. Les "Rondeaux" d'Anne de Graville, «Neophilologus», LXXXVIII, 2004, pp. 189202, notamment pp. 201-202. Pour le propos qui est le mien, le texte de Chartier transmis par le manuscrit fr. 2253 a systématiquement été comparé avec l'édition critique moderne procurée par J. Laidlaw. Cf. The poetical works of Alain Chartier cit.

(16) En reprenant les enseignements d'Aristote et d'Horace, dans l'Instructif, l'Infortuné avait souligné que récit et théâtre ne constituent que deux variantes de la même démarche créative: cf. C. THIRY, Le théatre, ou la poétique de l'entredeux cit., p. 58.

(17) E.J. CAYLEY, Debate and dialogue cit., pp. 144 et 148-149.

(18) Nous avons gardé la numérotation des huitains telle qu'elle apparaît dans le manuscrit transmettant la réécriture d'Anne de Graville; elle ne correspond pourtant pas à celle normalement attribuée par les éditeurs de la Belle dame sans mercy, car la poétesse ne réécrit pas la partie narrative du poème de Chartier, en se bornant à remanier le débat entre la dame et l'amant. 
occupent généralement (l'«endroit») (r. 67) permet d'atténuer la tonalité négative qui caractérise le personnage féminin dans le huitains; pareillement, l'allusion à la santé de l'amant, bien que réduite à «demy» (r. 45), diminue un peu sa détresse et, provenant «par raison» de la dame, rend le personnage masculin moins sujet à la domination arbitraire des personnages allégoriques.

Les stratégies de réécriture ne sont donc pas sans avoir des répercussions sur le sémantisme du texte, car les moindres changements linguistiques participent de la définition d'une nouvelle éthique courtoise. En effet, les enquêtes de C. Müller ${ }^{19}$ et $\mathrm{M}$. Bouchard ${ }^{20}$ ont démontré que la réécriture permet à la poétesse de présenter d'une façon détournée ses idées sur les relations entre les sexes ${ }^{21}$. Dans le milieu aulique du début du XVI ${ }^{\mathrm{e}}$ siècle, Anne de Graville crée des «personnages» qui s'expriment conformément à leur statut selon le principe de la congruentia illustré par l'Infortuné dans le Jardin de Plaisance ${ }^{22}$, et qui insistent à leur tour sur l'importance d'adapter l'ordre social à l'ordre du discours. C'est ainsi que dans le rondeau 24, la poétesse prête à la dame les mots suivants:

\section{[...] Selon les gens nous tenons le langaige, les extimans selon leur personnaige. \\ Ung foul parler sçavons baillir aux folz, aux importuns, les fascher de courroux et aux aymez nous faisons avantaige [...]} [r. 24, f. 14v]

La belle indifférente du texte de Chartier cède la place à une femme-éducatrice qui connaît les principes du decorum horatien ${ }^{23}$. Pareillement, le chevalier incarnant le malaise d'une société aristocratique marquée par la crise des valeurs courtoises ${ }^{24}$ se transforme en un homme à l'attitude modérée, qui doit suivre les encouragements de son interlocutrice dans le but de devenir un parfait courtisan ${ }^{25}$.

L'élaboration de ces «personnages» passe aussi par l'introduction de quelques interventions syntaxiques. Par exemple, dans le huitain 42, la dame refuse d'apprendre la discipline à laquelle elle devrait s'accoutumer, c'est-à-dire la façon de conforter le chevalier; au contraire, dans le rondeau correspondant, le verbe «aprendre» est adressé à l'amant: c'est lui qui doit retenir et appliquer le «mot exquis» conseillé par la femme, en se comportant honnêtement.

(19) C. MüLLER, Anne de Graville lectrice de "Maistre Allain": pour une récriture stratégique de la "Belle Dame sans Mercy", dans Lectrices d'Ancien Régime, éd. I. Brouard-Arends, Rennes, Presses Universitaires de Rennes, 2003, pp. 231-241.

(20) M. BOuCHARD, Les belles [in]fidèles cit.

(21) Elles ont notamment mis en évidence que l'adoption d'une rhétorique de l'exhortation, passant par la particularisation des énoncés, amoindrit la rigueur du débat de Chartier au profit d'une conversation plus équilibrée qui accentue l'importance du libre-arbitre des interlocuteurs. Cf. ibid., pp. 194-199.

(22) C. Thiry, Le théatre, ou la poétique de l'entredeux cit., p. 54; E. BuRon, "Faire en personnages" cit., pp. $215-216$.

(23) À propos de la connaissance et de l'interprétation du decorum horatien aux $\mathrm{XV}^{\mathrm{e}}$-XVI ${ }^{\mathrm{e}}$ siècle $\mathrm{cf}$. V. Leroux, Présence et interprétation du "decorum" horatien dans les poétiques néo-latines, «Camenae»13, 2012, pp. 1-15.

(24) D.F. Hult, La courtoisie en décadence. L'exemple de la "Belle Dame sans Mercy" de Alain Chartier, dans Progrès, réaction, décadence dans l'Occident médiéval, éd. E. Baumgartner, H. Harf-Lancner, Genève, Droz, 2003, pp. 251-260, notamment p. 259.

(25) D'après M. Bouchard, Anne de Graville se ferait promotrice de la «courtoisie aulique» ébauchée dans le Cortegiano de Castiglione, traité qui circula en France et en Italie dès 1516. Cette forme de courtoisie se distinguerait de la «courtoisie livresque», synonyme de «servitude», car elle correspondrait plutôt aux idées de «liberté» et «honneur». Cfr. M. BouCHARD, Les belles [in]fidèles cit., pp. 192-193. 


\begin{tabular}{|l|l|}
\hline \multicolumn{1}{|c|}{ Alain Chartier, La belle dame sans mercy } & \multicolumn{1}{|c|}{ Anne de Graville, Rondeaux } \\
\hline [...] Aultre confort donner ne sçay & [...] D'ung tel confort point ne me reprenez, \\
ne de l'aprendre n'ay je cure. & car aultre n'ay, pourtant le comprenez \\
Qui en veult en face l'essay. & et a l'aprendre y mettez soing et cure [...] \\
[h. 42, f. 22v] & [r. 40, f. 22v] \\
\hline
\end{tabular}

Dans le rondeau 59, le déplacement du mot-clé «cruaulté» cause un autre renversement sémantique par rapport au huitain. Dans le texte de Chartier, la «cruaulté» qualifie le déshonneur («le blasme et le desloz») qui tombera sur la dame si Pitié n'entrera pas dans son cœur; dans le rondeau correspondant, le mot décrit par contre les actions accomplies par la dame même, invitée à partager et distribuer («departez et si faictes le loz de cruauté») avec plus de clémence les souffrances d'amour: le blâme n'est donc plus imputable à un personnage allégorique extérieur, mais à la volonté de la femme («vous qui voulez»; «si vous voulez»), responsabilisée face à ses choix de vie.

\begin{tabular}{|l|l|}
\hline \multicolumn{1}{|c|}{ Alain Chartier, La belle dame sans mercy } & \multicolumn{1}{c|}{ Anne de Graville, Rondeaux } \\
\hline [...] Mais vous, qui si dur cueur portez & [...] Si vous voulez ung grant blasme emporter, \\
en si beau corps, si dire le os, & faictes qu'on puisse a chascun rapporter \\
gaignez $z^{26}$ le blasme et le desloz & que departez et si faictes les lotz \\
de crüaulté, qui mal y syet, & de cruaulté, qui ronge jusque a l'os \\
se Pitié qui depart les lotz & ung dolent cueur qui ne peult prouffiter \\
en vostre dur cueur ne se assiet. & de grant vertu $[\ldots]$ \\
[h. 61, f. 32r] & [r. 59, f. 32r] \\
\hline
\end{tabular}

Le débat entre le chevalier et sa dame se caractérise par le fait que chaque personnage cherche à se rapprocher le plus possible du point de vue de l'opposant pour s'en écarter ensuite ${ }^{27}$. Anne de Graville arrive même jusqu'à thématiser ce jeu de rôles dans les rondeaux. La présence d'allusions aux activités ludiques à l'intérieur du défi poétique constitue l'un des traits distinctifs des œuvres qui appartiennent à la «communauté collaborative» de la Querelle $e^{28}$. Dans cette réécriture, elles se manifestent sous la forme de développements visant à tisser un lien plus explicite entre la conquête de la dame et l'acte de jouer. La dimension du jeu représente d'ailleurs parfaitement la nature du chevalier et la dame ne manque pas de lui adresser des mots qui mettent en évidence cet aspect:

\begin{tabular}{|c|c|}
\hline Alain Chartier, La belle dame sans mercy & Anne de Graville, Rondeaux \\
\hline $\begin{array}{l}\text { Se aucun blesse aultruy d'aventure } \\
\text { sans coulpe de celluy qui blesse, } \\
\text { quoy qu'il n'en peult mais par droicture, } \\
\text { si en a il dueil et tristesse [...] } \\
{[\text { h. } 7, \text { f. } 5 \text { r] }}\end{array}$ & $\begin{array}{l}\text { S'il est ainsi que quelcun s'avanture } \\
\text { a ce jouer, et frappe d'avanture } \\
\text { si rudement que en ce jouant il blesse, } \\
\text { il en aura si grand deul et destresse } \\
\text { que le sien coeur en souffrira poincture }[\ldots . .] \\
{[\text { r. } 5, \text { f. } 5 \text { r }]}\end{array}$ \\
\hline
\end{tabular}

(26) Ms. Paris, BnF, fr. 2253, f. 31v: «gaigner».

(27) C. Müller, Anne de Graville lectrice de "Maistre Allain" cit., pp. 236-237.

(28) E.J. Cayley, Debate and dialogue cit., pp. 162-166. 
[...] Mon cueur pour aultruy ne hayray, pleure, crye, desconforte ou chante $[. .$. [h. 64, f. 33v]
[...] Vous plourerez, rirez, crirez, jourrez, et se voulez a voz maulx pourvoirez, mais pour aultruy mon cueur je ne hayray $[\ldots]$

[r. 62, f. 33v]

Dans la première citation, le verbe «jouer», absent du huitain de Chartier, figure deux fois dans le rondeau, où il est précédé du démonstratif «ce» permettant de souligner l'identité entre la légèreté d'un passetemps et l'imprudence avec laquelle l'amant s'aventure dans son entreprise galante. Dans le deuxième extrait, la poétesse décrit les actions de l'amant par ce même verbe, et non pas par «chanter», comme l'avait fait Chartier dans son texte, en laissant entendre que la nature des divertissements n'est pas précisée et qu'elle pourrait bien se référer tant à des activités ludiques qu'à des conquêtes amoureuses ${ }^{29}$.

Comme il l'a été évoqué ci-dessus, le jeu des rôles qui caractérise le débat amoureux évoque aussi un autre espace fictionnel, celui du théâtre:

\begin{tabular}{|c|c|}
\hline Alain Chartier, La belle dame sans mercy & Anne de Graville, Rondeaux \\
\hline $\begin{array}{l}\text { Dames ne sont mye si lourdes, } \\
\text { si mal entendans, ne si folles, } \\
\text { que pour ung peu de plaisans bourdes } \\
\text { confites en belles parolles, } \\
\text { dont vous aultres tenez escolles } \\
\text { pour leur faire croyre merveilles, } \\
\text { qu'elles changent si tost leurs colles: } \\
\text { a beau parler closes oreilles. } \\
\text { [h. } 14, \text { f. } 8 \mathrm{v}]\end{array}$ & $\begin{array}{l}\text { Quoy qu'en pensez, dames ne sont si folles } \\
\text { que pour ung peu de fringantes parolles, } \\
\text { de fainct semblant et de plaisantes bourdes } \\
\text { on les deçoipve ainsi qu'on feroit lourdes } \\
\text { pour se laisser tromper en chauldes colles. } \\
\text { Ilz sçaivent bien que c'est que de frivolles } \\
\text { et qu'en tenez les publicques ecolles, } \\
\text { mais ce ne sont bagues ny hapelourdes, } \\
\text { quoy qu'en pensez. } \\
\text { Chacun congnoist que avez bons protocolles } \\
\text { de fainctz parlers que estudiez par rolles } \\
\text { comme menteurs qui n'ont les langues gourdes; } \\
\text { mais ilz auront toujours oreilles sourdes } \\
\text { a beau parler et fines parabolles, } \\
\text { quoy qu'en pensez. } \\
\text { [r. } 12, f \text { f. } 8 \text { v }\end{array}$ \\
\hline
\end{tabular}

Dans ce rondeau, Anne de Graville retient l'opposition entre les séducteurs et les dames chastes ébauchée par Chartier; elle décide néanmoins de développer le sujet à l'aide d'amplifications portant sur l'univers du théâtre. En comparant la fiction de la représentation scénique aux mensonges des hommes engagés dans la conquête amoureuse, la dame décrit l'amant comme un acteur caractérisé par un «fainct semblant», qui étudie les formules («protocolles») des «fainctz parlers» écrits dans des «rolles», mot qu'on sait désigner le support contenant la transcription de ce que l'on a à réciter dans une pièce de théâtre ${ }^{30}$. Toutefois, la dame ne l'écoute pas: elle n'est pas une femme facile et elle n'aime pas tromper («ce ne sont bagues ny hapelourdes»). L'emploi du vocable «bagues», normalement utilisé dans des contextes théâtraux comiques ou satiriques pour indiquer les femmes galantes ${ }^{31}$, révèle que la poétesse

(29) DMF 2015, «jouer», A1.

(30) DMF 2015, «role», B.

(31) DMF 2015, «bague 3». 
attribue une attention particulière au réseau lexical du théâtre. À ce propos, ce n'est peut-être pas un hasard qu'elle décide aussi d'employer à deux reprises le pronom sujet neutre «ilz» pour le féminin. Bien qu'au XVI ${ }^{e}$ siècle le système des pronoms personnels soit celui de la langue moderne, cet emploi bien attesté en moyen français est encore témoigné par les textes de la première moitié du siècle; dans ce rondeau, il pourrait notamment constituer une référence aux farces qui, selon les relevés effectués par S. Lardon et M.-C. Thomine, en seraient particulièrement riches ${ }^{32}$.

À l'imitation du jeu d'échecs, construit sur le modèle de la bataille, les modalités $\mathrm{du}$ jeu amoureux dans les rondeaux se traduisent parfois aussi dans des images de guerre, dans lesquelles les stratégies de conquête évoquent les tactiques de combat:

\begin{tabular}{|c|c|}
\hline Alain Chartier, La belle dame sans mercy & Anne de Graville, Rondeaux \\
\hline $\begin{array}{l}\text { [...] Se moy ou aultre vous regarde, } \\
\text { les yeulx sont faiz pour regarder. } \\
\text { Je n'y prens point aultrement garde: } \\
\text { qui mal y sçet s'en doit garder }[. . .] \\
\text { [h. } 6, \text { f. } 4 \mathrm{v}]\end{array}$ & $\begin{array}{l}\text { [...] Qui mal y fait s'en doit contregarder } \\
\text { et en l'assault se taudir et barder } \\
\text { en se tenant en tout temps sus sa garde. } \\
\text { Qui danger crainct que bien se contregarde } \\
\text { mais point ne fault son dire ainsi farder }[\ldots] \\
{[\text { r. } 4, f .4 \mathrm{v}]}\end{array}$ \\
\hline
\end{tabular}

Dans ce rondeau, Anne de Graville introduit le topos du siège militaire dans la tentative d'amoindrir la sévérité du reproche adressé par la dame à son prétendant. En effet, chez la poétesse, les mots de la femme invitent l'amant à se mettre à l'abri des assauts de l'amour. Le foisonnement des termes qui renvoient au champ sémantique de la défense («contregarder», «taudir», «barder») semble comparer l'âme du chevalier à une ville en danger qui doit se protéger («se tenant en tout temps sus sa garde»). D'ailleurs, la réécriture est parsemée de références aux moments de la guerre qui ne font pas partie du texte de Chartier: l'embuscade («mais Faulx Semblant, le trahistre en contenance, l est en aguet puis nostre aage d'enfance» [r. 68, f. 36v]), le siège («deul et ennuy mon povre coeur assault» [r. 1, f. 3r]), la conquête («et ne cuidez vous ou amant aucun | gaigner ung coeur pour faire l'importun» [r. 16, f. 10v]), la paix («la paix enseigne en quoy nuyst guerre dure» [r. 15, f. 10r]).

Par delà la thématisation du jeu, E. Cayley a également démontré qu'à l'intérieur de la «communauté collaborative» des continuateurs de la Belle dame sans merci, les textes nouent des liens entre eux: chaque réélaboration ne se borne pas à emprunter la matière littéraire au chef d'œuvre de Chartier, mais elle s'inspire aussi des textes de la Querelle dans une confrontation plus étendue ${ }^{33}$. Par exemple, dans le remaniement d'Anne de Graville, on peut déceler quelques points en commun avec d'autres réécritures de la Belle dame sans mercy. Le chevalier de la poétesse rappelle le «pauvre amant» de L'amant rendu cordelier, ouvrage classé parmi les représentants du troisième cycle de la Querelle et datant de $1440^{34}$. Tous les deux prétendants aiment les rendez-vous mondains, mais ils se voient soudainement obligés à renoncer à la poésie et aux chansons:

(32) S. Lardon, M.-C. Thomine, Grammaire du français de la Renaissance. Étude morphosyntaxique, Paris, Classiques Garnier, 2009, p. 75.

(33) E. J. CaYley, Debate and dialogue cit., pp. 156-157.

(34) Ibid., p. 145. 


\begin{tabular}{|c|c|c|}
\hline $\begin{array}{c}\text { Alain Chartier, } \\
\text { La belle dame sans mercy }\end{array}$ & Anne de Graville, Rondeaux & L'amant rendu cordelier \\
\hline $\begin{array}{l}\text { Je ne suis mye bon chanteur, } \\
\text { aussy me duyt myeulx le } \\
\text { plourer, } \\
\text { mais je ne fus oncques } \\
\text { vanteur }[\ldots]\end{array}$ & 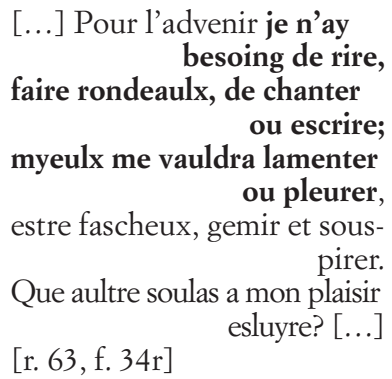 & $\begin{array}{l}\text { [...] Quant menestriés vous } \\
\text { orrez, felutes, doucines ou } \\
\text { vielles, } \\
\text { vous grain ne les escouterés, } \\
\text { mais metrés voz dois aux } \\
\text { oreilles }^{35}[\ldots] \\
{[\text { vv. 1389-92] }}\end{array}$ \\
\hline
\end{tabular}

Anne de Graville renverse visiblement le texte de Chartier, car son chevalier sait chanter et composer des poèmes; en revanche, le jeune homme décrit dans le rondeau doit renoncer à ses divertissements, comme le protagoniste de L'amant rendu cordelier. La différence substantielle entre ce texte et le rondeau consiste dans la source de l'interdiction. C'est «Damp Prieur», un personnage secondaire, qui invite «l'amant rendu cordelier» à se boucher les oreilles, tandis que c'est le libre-arbitre qui impose la tristesse à l'amant des Rondeaux. Au moyen de cette intervention personnelle, la poétesse saisit donc l'occasion d'insister sur la volonté personnelle du chevalier.

Enfin, un autre élément qui permet de mettre en rapport ce remaniement avec la «communauté collaborative» de la Querelle consiste dans l'épilogue de l'ouvrage. La Belle dame sans mercy se termine avec les mots du poète-narrateur qui avait écouté le débat entre le chevalier et sa dame: il reprend le récit et raconte qu'on lui a appris la mort de l'amant; ensuite, il donne des conseils aux amoureux et prie les dames de ne pas être trop cruelles. On sait que les réécritures de la Querelle se caractérisent généralement par des conclusions ouvertes. L'œuvre de la poétesse ne fait pas exception sur ce point: dans le dernier rondeau, le narrateur annonce que l'amant abandonne les lieux de la fête en proie à un désespoir mortel; bien que le «je» poétique se lance à la poursuite du jeune homme désabusé pour essayer de le consoler, il n'y parvient pas et il ne sait pas non plus ce qui lui arrivera:

\section{[...] Je luy voulluz lorz estre secourant et tout souldain je vins vers luy courant, mais je ne sçeuz qu'il devint a l'apresse ainsi partit. \\ On me dist bien qu'il s'en alloit tirant} tous ses cheveulx et qu'il alloit mourrant par grant courroux et extresme tristesse, et que aucun deuil n'en a pris sa maistresse, combien qu'il fust par griefz plaintz souspirant [...]

$$
\text { [r. 71, f. 38r] }
$$

Anne de Graville se conforme au topos de l'incertitude narrative et renonce à fournir un message moral. Elle ne réécrit pas les derniers huitains de Chartier adressés aux amoureux et aux dames: sa voix poétique reste neutre. D’après E. Cayley, la 
suspension de la conclusion correspondrait au désir de perpétuer la création poétique et tout épilogue provoquerait l'écroulement des dynamiques sur lesquelles se fonde la survivance de la «communauté collaborative» de la Querelle $e^{36}$. La poétesse semble donc participer activement aux échanges littéraires, en laissant ouvert son ouvrage afin que d'autres écrivains puissent relever le défi et s'inscrire au sein du même réseau intellectuel.

Presque cent ans séparent la rédaction de la Belle dame sans mercy du remaniement élaboré par Anne de Graville; pendant ce temps, les goûts littéraires des lecteurs et la notion même de «courtoisie» ont évolués ${ }^{37}$ L'activité poétique à la cour de François $I^{\text {er }}$ est plus qu'un passe temps ou une forme raffinée de galanterie: le débat amoureux thématisé dans la pratique littéraire est conçu comme un moyen pour accompagner les ambitions politiques du jeune roi se voulant affirmer en prince et en poète. La dame d'honneur de la reine Claude ne renonce donc pas à se confronter avec «maistre Allain» à la lumière d'un jugement critique renouvelé; le choix du rondeau ne se justifie pas uniquement par une préférence pour l'effet circulaire et les sonorités musicales des refrains ${ }^{38}$, car la poétesse semble partager avec l'auteur de l'Instructif de seconde rhetorique l'admiration pour les potentialités théâtrales de cette forme poétique ${ }^{39}$. La mise en page du manuscrit, ainsi que les procédés de réécriture portent au premier plan le souhait de mettre en scène une pluralité de voix qui correspondent à des personnages bien caractérisés du point de vue de l'appartenance sociale et de l'élocution. De plus, la cohésion entre la forme et le contenu du remaniement est renforcée par la multiplication d'images ludiques qui évoquent le jeu de rôles entre le chevalier et la dame, et qui nous permettent d'inscrire les Rondeaux à l'intérieur d'une «communauté collaborative» virtuelle, dont le but est celui de s'agrandir en inspirant plusieurs générations de poètes.

(38) C. Müller, Anne de Graville lectrice de "Maistre Allain" cit., p. 232.

(39) C. Thiry, Le théatre, ou la poétique de l'entredeux cit., p. 50. 